\title{
Utility Greedy Discrete Bit Loading for interference limited Multi-Cell OFDM system
}

\author{
Xinghui Su, Tiejun Lv, Member, IEEE, Xuefen Yu, Shaoshi Yang, Yonghua Li \\ Key Lab of Universal Wireless Communications, Ministry of Education, \\ Beijing University of Posts and Telecommunications, Beijing, China 100876
}

\begin{abstract}
In this contribution we present the solution of the utility greedy discrete bit loading for interference limited multicell OFDM networks. Setting the utility as the sum of consumed power proportions, the algorithm follows greedy way to achieve the maximum throughput of the system. Simulation has shown that the proposed algorithm has better performance and lower complexity than the traditional optimal algorithm. The discussion of the results is provided .
\end{abstract}

\section{INTRODUCTION}

Cooperative resource allocation is an effective method to avoid the inter-cell interference in multi-cell OFDMA systems. Resource allocation for OFDMA networks has three major tasks: subchannel assignment, rate allocation, and power control. Since the inter-cell interferences are affected by the subchannel assignment, the corresponding rate adaptation and power control, any change of the resource allocation in a specific cell will affect the other nearby cells. Thus, the cooperative resource allocation is a very complex process in the multi-cell OFDMA systems. This paper only considers the situation when the subchannel allocations have been done. More specifically, this paper considers the cooperative resource allocation in the multi-cell OFDM systems.

The relevant researches could be found in the fields of DSL systems, cognitive radio networks [1], [2], and [3]. The optimal spectrum balancing (OSB) algorithm has been shown in [4]. The OSB algorithm converts the maximum problem to the weighted problem and employs the dual decomposition to solve it. The iterative spectrum balancing algorithm which follows the idea of OSB is proposed and obtains much smaller complexity [5], [6]. However, the weighted optimization needs to predetermine each user's weight and their target rate, which makes the optimized result can't be obtained directly. Thus, the weighted optimization algorithms has to check all the possible weight combination in order to get the maximum, which make the algorithm become too complex. Further more, there are some internal disadvantages for OSB that could bring performance degradation. These disadvantages will be discussed in the follows.

This research has been supported by Program for New Century Excellent Talents in University (NCET) under Grant No. NCET-06-0091, National Natural Science Foundation of China (NSFC) under Grant No.60972075, Specialized Research Fund for the Doctoral Program of Higher Education (SRFDP) grant No. 20070013018 and the Fundamental Research Funds for the Central Universities.

Xinghui Su, Tiejun Lv (xhui.su@gmail.com, lvtiejun@tsinghua.org.cn)
Our work invokes the greedy method and provides the utility greedy discrete bit loading for the interference limited multicell OFDM systems. The simulation results prove that the optimal discrete bit loading algorithm performs better than OSB. Further more, the utility greedy discrete bit loading has much smaller complexity than OSB.

\section{System ModeL}

We consider an uplink multiuser OFDMA Multi-Homecell system. Suppose that there are $N(N \geq 2)$ pairs of transmitter and receiver, each of them occupies the same span of tones and is equipped with one transmit and one receive antenna. We assume that one receiver gets desired information from one predetermined transmitter. Then the received signal-tointerference-plus-noise ratio (SINR) for the $i_{t h}$ receiver at tone $k$ is

$$
\operatorname{SINR} R_{i}^{[k]}=\frac{p_{i}^{[k]} g_{i, i}}{\sum_{j=1, j \neq i}^{N} p_{j}^{[k]} g_{i, j}+\sigma},
$$

where $p_{i}^{[k]}$ is the transmitted power spectrum density (PSD) at tone $k$ by the $i_{t h}$ transmitter, $g_{i, j}$ is the channel gain from the $j_{t h}$ to the $i_{t h}$ receiver and $\sigma$ is the additive, white and Gaussian noise (AWGN) PSD.

The goal of this paper is to maximize system's throughput by allocating power for each transmitter at each tone

$$
\begin{aligned}
\max _{\vec{P}} & \sum_{i=1}^{N} \sum_{k=1}^{K} r_{i}^{[k]} \\
\text { s.t. } & \sum_{k=1}^{K} p_{i}^{[k]} \leq P_{i, \max }, \forall i,
\end{aligned}
$$

where $P_{i, \max }$ is the total power constraint for the $i_{t h}$ transmitter and $r_{i}^{[k]}$ is the achievable bit loading of the $i_{t h}$ receiver on tone [7]. $r_{i}^{[k]}$ is obtained by

$$
r_{i}^{[k]} \triangleq \log _{2}\left(1+\frac{1}{\Gamma} S I N R_{i}^{[k]}\right),
$$

where $\Gamma$ denotes the SNR-gap to capacity, which is a function of the desired BER, coding gain and noise margin. Formulation (3) could be rewritten as

$$
p_{i}^{[k]}-\Gamma\left(2^{r_{i}^{[k]}}-1\right) \sum_{j=1, j \neq i}^{N} p_{j}^{[k]} \frac{g_{i, j}}{g_{i, i}}=\Gamma\left(2^{r_{i}^{[k]}}-1\right) \frac{\sigma}{g_{i, i}}
$$


And since the modem supports only integer bitloading, the search space could be reduced according to exact bitloadings. Thereby these can be written in matrix form as

$$
[\mathrm{I}-\Psi G] P=\Psi \Omega
$$

$$
\begin{aligned}
& \text { where } \mathbf{P}_{N \times 1}= \\
& \Omega_{N \times 1}=\left[\begin{array}{ccc}
p_{1}^{[k]} & \cdots & p_{N}^{[k]}
\end{array}\right]^{T}, \\
& \operatorname{diag}\left\{\frac{\Gamma\left(2^{r_{1}^{[k]}}-1\right)}{g_{1,1}}, \ldots, \frac{\Gamma\left(2^{r k}-1\right)}{g_{N, N}}\right\} \text { and } G_{N \times N}= \\
& {\left[\begin{array}{cccc}
0 & g_{1,2} & \cdots & g_{1, N} \\
g_{2,1} & 0 & \cdots & g_{2, N} \\
\vdots & \vdots & \ddots & \vdots \\
g_{N, 1} & g_{N, 2} & \cdots & 0
\end{array}\right] .}
\end{aligned}
$$

Then this cooperative resource allocation problem on tone $k$ is feasible for all simultaneous transmitting-receiving pairs as long as the matrix $(\mathrm{I}-\Psi \cdot G)$ is non-singular, i.e. invertible. Then the power allocated for $r_{k}$ is

$$
P=[\mathrm{I}-\Psi G]^{-1} \Psi \Omega .
$$

This simple power allocation scheme, however, suffers from large complexity $O\left(\left(b_{\max }+1\right)^{N K}\right)$, where $b_{\max }$ is the maximal bitloading that one modem could support. Considering and $\mathrm{K}$ is large in OFDMA system, this problem becomes intractable. The OSB algorithm below reduces this complexity to some extent. And Utility Greedy Discrete Bit Loading algorithm we proposed further edges that complexity.

\section{UTILITY GREEDY DISCRETE BIT LOADING}

This work provides the utility greedy discrete solutions when such constraints are taken into account. For each tone $k(1 \leq k \leq K)$, we assume that the sum of bit loading $R^{[k]}$ could be transmitted using power function $e_{k}\left(R_{K}\right) \triangleq$ $\left[e_{1}^{[k]}, \ldots, e_{N}^{[k]}\right]$. Actually, there are numerous $r_{k}$ which could satisfy $R^{[k]}=\sum_{i=1}^{N} r_{i}^{[k]}$ while under the power constraint. Define the function $\gamma$ as

$$
\gamma\left(R^{[k]}\right)=\left\{r_{k} \mid \sum_{i=1}^{N} r_{i}^{[k]}=R^{[k]}, r_{i}^{[k]}<r_{\max }, k=1 \ldots K\right\}
$$

where $r_{\max }$ is the maximum bit loading constrained by the system . If $r_{k}$ is determined and available $\left(\mathbf{I}-\boldsymbol{\Lambda}_{\mathbf{k}} \mathbf{G}\right)$ is invertible), the power function $e_{k}\left(R_{K}\right)$ could be calculated as

$$
e_{k}\left(R^{[k]}\right)=p_{k}=\left(\mathbf{I}-\boldsymbol{\Lambda}_{\mathbf{k}} \mathbf{G}\right)^{-\mathbf{1}} \boldsymbol{\Lambda}_{\mathbf{k}} \sigma .
$$

Introduced the incremental power function

$$
\begin{aligned}
\Delta e_{k}\left(R^{[k]}\right) & \triangleq\left[\Delta e_{1}^{[k]}, \ldots, \Delta e_{N}^{[k]}\right] \\
& =\left\{\begin{array}{c}
e_{k}\left(R^{[k]}\right)-e_{k}\left(R^{[k]}-1\right), \text { if } R^{[k]} \geq 1 \\
e_{k}\left(R^{[k]}\right), \text { if } 0 \leq R^{[k]} \leq 1 .
\end{array}\right.
\end{aligned}
$$

Suppose the remaining power to be allocated for each pair is $\Delta P_{1}, \ldots \Delta P_{N}$ when the sum of bit loading on tone $k$ is

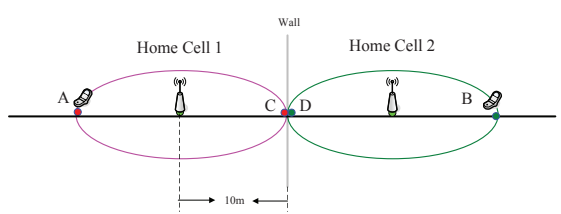

Fig. 1. Homecell simulation environment

$R^{[k]}-1$. Then when the sum of bit loading increases to $R^{[k]}$, the utility function is set as

$$
u\left(R^{[k]}\right)=\sum_{i=1}^{n} \frac{\Delta e_{i}^{[k]}}{\Delta P_{i}}
$$

The proposed optimal discrete bit loading algorithm is as follows:

\section{Initialization}

Initialize $r_{k} \triangleq[0, \ldots, 0]^{T}, F \triangleq[0, \ldots, 0]^{T}$ for $k=1, \ldots, K$. $\{F\}_{k}=1$ is the flag which indicates the feasibility of adding more bits to the tone $k$ under the power constraint, whereas $\{F\}_{k}=0$ means that the tone $k$ is saturated. Let $p_{k} \triangleq\left[p_{1}^{[k]}, \ldots, p_{N}^{[k]}\right]$. The total bit loading of the system is initialized as $R=\sum_{k=1}^{K} R^{[k]}=0$.

\section{Bit loading Iteration:}

Step 1. Check the possibility to add one more bit to the total bit loading of the system.

1). Check that if it is possible to add one more the bit loading to each tone. For the tone $k$,

a) Generate the available $r_{k}$ set when add one more bit as $\gamma\left(R^{[k]}+1\right)$. For each $r_{k} \in \gamma\left(R^{[k]}+1\right)$, calculate the $e_{k}\left(R_{K}\right)$ using (12). If the $e_{k}\left(R_{k}+1\right)$ satisfies the power constraint, which could be denoted as $\sum_{q=1, q \neq k}^{K}\left(p_{i}^{[q]}+\left\{e_{k}\left(R_{k}+1\right)\right\}_{i}\right) \leq$ $P_{i, \max }, i=1 \ldots N$, set $\{F\}_{k}=1$.

b) Search for $r_{k}^{*}=\underset{r_{k}^{*} \in \gamma\left(R^{[k]}+1\right)}{\arg } \min \left\{u\left(R^{[k]}+1\right)\right\} \quad$ and record the relevant $e_{k}\left(R_{k}+1\right)$ and utility $u\left(R^{[k]}+1\right)$. 2). If $F$ equals to $[0, \ldots, 0]^{T}$, stop the iteration. On the contrary, go to Step 2.

Step 2. Updating the bit loading.

1). Collect all the subchannels that could be added to one more bit as $\nu=\left\{k \mid\{F\}_{k}=1, k \in\{1, \ldots, K\}\right\}$.

2). Search for $k^{*}=\underset{k \in \nu}{\arg \min }\left\{u\left(R^{[k]}+1\right)\right\}$.

3). Let $r_{k^{*}}=r_{k^{*}}^{*}$ and $p_{k^{*}}=e_{k^{*}}\left(R_{k^{*}}+1\right)$. Thus, $R_{k^{*}}=$ $R_{k^{*}}+1$ and $R=R+1$, which means the total bit loading increases by one bit.

4) Re-initialize $F \triangleq[0, \ldots, 0]^{T}$ for $k=1, \ldots, K$.

5). Go to Step1.

Note that the complexity of the utility greedy discrete bit loading algorithm (UG) is $V_{U G}=O\left(K^{2} \sum_{b=1}^{b_{\max }+1} b^{N}\right)$.

\section{Simulation And Discussion}

The utility greedy discrete bit loading algorithm is numerical simulated using the channel model of homecells. 
TABLE I

HOMECELl SimUlation PARAMETERS

\begin{tabular}{|c|c|}
\hline \multicolumn{1}{|c||}{ Parameters } & Value \\
\hline Cell radius & $10 \mathrm{~m}$ \\
\hline Antenna gain & $0 \mathrm{dBi}$ \\
\hline UE antenna gain & $0 \mathrm{dBi}$ \\
\hline Distance dependent pathloss & $39+20 \log (\mathrm{r})+10 \mathrm{n} \mathrm{dB}$ \\
\hline Tx power & {$[-10,20] \mathrm{dBm}$} \\
\hline Bandwith (W) & $100 \mathrm{MHz}$ \\
\hline Subcarrier bandwidth & $15 \mathrm{KHz}$ \\
\hline Noise power on each subcarrier & $-174 \mathrm{dBm} / \mathrm{Hz}$ \\
\hline Noise Figure & $9 \mathrm{~dB}$ \\
\hline
\end{tabular}

Referred to TR25.814 and R1-084026 of 3GPP, the simulation parameters are shown in TABLE I.

The two homecells model is invoked for the simulations of this section. Assume that it contains only one user in each homecell. A wall exists between the two homecells which is a very common scene in the office building. Then the simulation environment is shown as Figure 1.

In order to evaluate the performance of utility greedy discrete bit loading algorithm, the comparison with the OSB would be given. Because of the limit of conditions and huge complexity of OSB, this paper only compares the performance of both algorithms in three typical pairs of locations, e.g. when the users of Homecell 1 and Homecell 2 locate respectively in $\mathrm{A}$ and $\mathrm{B}, \mathrm{A}$ and $\mathrm{D}, \mathrm{C}$ and $\mathrm{D}$. When the users of the two home cells locate in $\mathrm{A}$ and $\mathrm{B}$, the interference for both two users is very small. When the users of the two home cells locate in $\mathrm{A}$ and $\mathrm{D}$, the interference for user in home cell 1 is small while the interference for user in home cell 2 is large. When the users of the two home cells locate in C and D, the interference for both users are large. The simulations in these three pairs of locations could fully reveal the comparison the utility greedy discrete bit loading algorithm (UG Algorithm) and optimal spectrum balancing algorithm (OSB Algorithm). Suppose that the two homecells utilize the same TX powers. The total throughput of the system with different TX power is depicted in Figure 2, Figure 3 and Figure 4.

It's obvious that the utility discrete bit loading algorithm always behaves better performance than the optimal spectrum balancing algorithm. The reason will be discussed in the next section. Considering the complexity of UG, it is easily known that the UG discrete bit loading algorithm has much smaller complexity than the optimal spectrum balancing algorithm.

\section{Results' Discussion}

The simulation results would be discussed in this section. It is not difficult to discover that the curve of the OSB algorithm maintains on a value for some TX powers in Figure2 and Figure3. Actually, this phenomenon is caused by some disadvantage of the OSB algorithm. In order to make it clear, we first introduce the Lemma1.

Lemma1: In order to maximize the throughput of the system, the two pairs $i, j$ should share the tone $k(k=1, \ldots, K)$

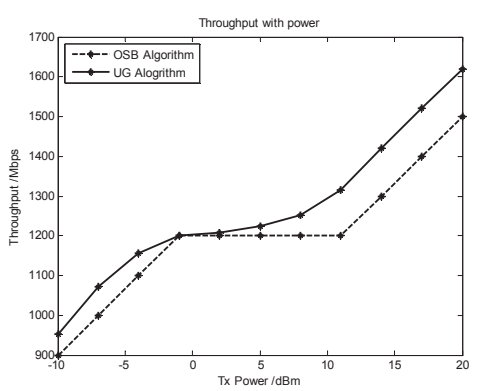

Fig. 2. Users of Home Cell 1 and Home Cell 2 loacte in A and B respectively.

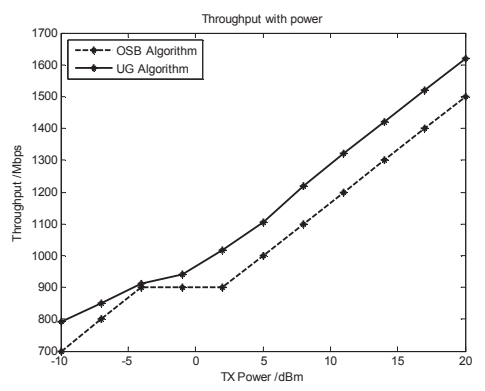

Fig. 3. Users of Home Cell 1 and Home Cell 2 loacte in A and D respectively.

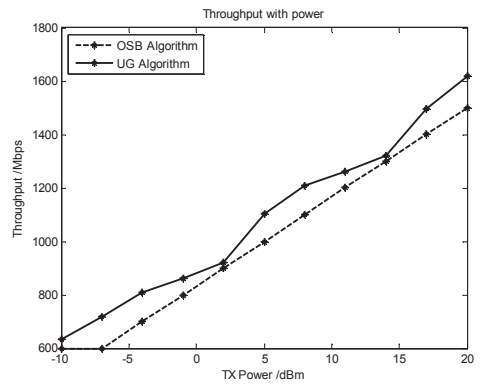

Fig. 4. Users of Home Cell 1 and Home Cell 2 loacte in C and D respectively.

simultaneously when satisfy

$$
\frac{p_{i}^{[k]} g_{i, i}}{p_{j}^{[k]} g_{i, j}} \geq \frac{p_{j}^{[k]} g_{i, j}}{Z_{i}^{[k]}}, \quad i, j=1, \ldots, N
$$

where $Z_{i}^{[k]}=\sigma+I_{i}^{[k]}, I_{i}^{[k]}$ is the interference for pair $i$ on tone $k$.

Otherwise, the pairs $i, j$ should not transmit on the tone $k$ simultaneously when

$$
\frac{p_{i}^{[k]} g_{i, i}}{p_{j}^{[k]} g_{i, j}}<\frac{p_{j}^{[k]} g_{i, j}}{Z_{i}^{[k]}}, \quad i, j=1, \ldots, N .
$$

The proof of Lemmal could be found in Appendix.

With Lemma1, the cooperative resource allocation problem could be divided into three states: spectrum sharing state, spectrum partitioned state and spectrum mixed state. The definitions of each state are describe as follows. 
Spectrum sharing state, which is defined as all the pairs share all the tones simultaneously.

Spectrum partitioned state, which is defined as all the tones are only occupied by one pair at a time.

Spectrum mixed state, which is defined as that some tones are utilized by only one pair while others are shared by different pairs.

The OSB algorithm drops its optimum when the dual problem is decomposed into a set of sub-problems. After the decomposition, the cooperative optimization on different tones couldn't be done which cause the performance degradation. Particularly, when the channel gain is considered in this paper, a pair has the same channel station on all the tones. Then the OSB algorithm is only effective on the spectrum sharing state and spectrum partitioned state. When the TX power is in the field of the spectrum mixed state, the OSB algorithm only stays at a value which is the maximum in the spectrum sharing state. Thus the curve of the OSB algorithm always maintains on a value for some TX powers in Figure2 and Figure3.

\section{Proof:}

In the spectrum partitioned state, the tone $k$ is only occupied by a pair of transmitter and receiver. Assume the pair $i$ occupy the tone when the bit loading is $R_{k}$, then

$$
R_{k}=r_{i}^{[k]}=\log _{2}\left(1+\frac{1}{\Gamma} \frac{p_{i}^{[k]} g_{i, j}}{\sigma}\right) .
$$

The power need to allocated by pair $i$ is

$$
p_{i}^{[k]}=\frac{\left(2^{R_{k}}-1\right) \Gamma \sigma}{g_{i, j}} .
$$

Suppose the $R^{[k]}+1$ is achievable on tone $k$. When $2^{R_{k}} \gg$ 1 , the utility could be obtained as

$u\left(R_{k}+1\right)=\left\{\begin{array}{lc}\frac{p_{i}^{[k]}\left(R_{k}\right)}{\left[\Delta P_{i}\right.} & \text { if } r_{i}^{[k]}=R_{k}+1 \\ \frac{p_{j}^{[k]}\left(R_{k}+1\right)}{\Delta P_{j}}-\frac{p_{i}^{[k]}\left(R_{k}\right)}{\Delta P_{i}} & \text { if } r_{j}^{[k]}=R_{k}+1, j \neq i\end{array}\right.$

Compare the different result of (20),

$$
\frac{p_{j}^{[k]}\left(R_{k}+1\right)}{\Delta P_{j}}-\frac{p_{i}^{[k]}\left(R_{k}\right)}{\Delta P_{i}}-\frac{p_{i}^{[k]}\left(R_{k}\right)}{\Delta P_{i}} \approx \frac{2^{\Delta r_{i}^{[k]}}-2^{\Delta r_{j}^{[k]}}}{2^{\Delta r_{j}^{[k]}+\Delta r_{i}^{[k]}-1}},
$$

where $\Delta r_{j}^{[k]}$ is the bit loading could be added on tone $k$ potentially. It is obvious that the minimum of $u\left(R_{k}+1\right)$ will choose the pair who could obtain the most bit loading. According to [8], [9], it is optimum when choose the minimum $u\left(R_{k}+1\right)$. Thus, the utility greedy discrete bit loading algorithm is the optimal algorithm in the spectrum partitioned state.

\section{CONCLUSION}

In this paper, we propose the utility greedy discrete bit loading algorithm for the interference limited multi-cell OFDM systems. And three kinds of spectrum states are proposed. The UG algorithm is also proved to be the optimal algorithm in the spectrum partitioned state. In our next research, we go on searching for the optimal algorithm for the interference limited multi-cell OFDM systems as well as the multi-cell OFDMA systems.

\section{APPENDIX}

\section{Proof of Lemma1:}

Suppose two pairs $i, j$ could transmit their data on tone $k$ and $l$. Assume that these two tones has the same channel state, then $Z_{i}^{[k]}=Z_{i}^{[L]}, Z_{j}^{[k]}=Z_{j}^{[L]}$. When they both transmit their data simultaneously on the two tones, the PSD of each pair on each tone is $p_{i}^{[k]}$ and $p_{j}^{[k]}$ respectively. The total bit loading is

$$
\begin{aligned}
R_{k+l} & =2 \log _{2}\left(1+\frac{1}{\Gamma} \frac{p_{i k}^{[k]} g_{i, j}^{[k]}}{p_{i}^{[k]} g_{i, j}^{[k]}+Z_{i}^{[k]}}\right) \\
& +2 \log _{2}\left(1+\frac{1}{\Gamma} \frac{p_{j}^{[k]} g_{j, j}^{[k]}}{p_{i}^{[k]} g_{j, i}^{[k]}+Z_{j}^{[k]}}\right) .
\end{aligned}
$$

Otherwise, when the each pair transmits it data on its unique tone, the total bit loading become $R_{k+l}^{\prime}=$ $\log _{2}\left(1+\frac{1}{\Gamma} \frac{2 p_{i}^{[k]} g_{i, i}}{Z_{i}^{[k]}}\right)+\log _{2}\left(1+\frac{1}{\Gamma} \frac{2 p_{j}^{[k]} g_{j, j}}{Z_{j}^{[k]}}\right)$.

In order to compare these two data rate, let $R_{k+l}$ minus $R_{k+l}^{\prime}$, we have

$$
\begin{aligned}
& R_{k+l}-R_{k+l}^{\prime} \quad S I N R \gg \Gamma, p g \gg Z \\
& \log _{2}\left(\frac{p_{i}^{[k]} g_{i, i} /\left(p_{i}^{[k]} g_{i, j}\right)}{p_{i}^{[k]} g_{i, j} / Z_{i}^{[k]}}\right)+\log _{2}\left(\frac{p_{j}^{[k]} g_{j, j} /\left(p_{i}^{[k]} g_{j, i}\right)}{p_{i}^{[k]} g_{j, i} / Z_{j}^{[k]}}\right) .
\end{aligned}
$$

As seen from the above formulation, the two pairs should transmit data on tone $k$ simultaneously in order to achieve the maximum bit loading when $\frac{p_{i}^{[k]} g_{i, i}}{p_{j}^{[k]} g_{i, j}} \geq \frac{p_{j}^{[k]} g_{i, j}}{Z_{i}^{[k]}}$ and $\frac{p_{j}^{[k]} g_{j, j}}{p_{i}^{[k]} g_{j, i}} \geq$ $\frac{p_{i}^{[k]} g_{j, i}}{Z_{j}^{[k]}}$. Otherwise, when $\frac{p_{i}^{[k]} g_{i, i}}{p_{j}^{[k]} g_{i, j}}<\frac{p_{j}^{[k]} g_{i, j}}{Z_{i}^{[k]}}$ and $\frac{p_{j}^{[k]} g_{j, j}}{p_{i}^{[k]} g_{j, i}}<$ $\frac{p_{i}^{[k]} g_{j, i}}{Z_{j}^{[k]}}$, the two pairs should be frequency orthogonal to achieve the maximum data rate.

\section{REFERENCES}

[1] L. Cao, L. Yang, and H. Zheng, "The impact of frequencyagility on dynamic spectrum sharing," in Proc. of IEEE DySPAN, 2010.

[2] C. Raphael, J. Huang, C. Mung, and M. Marc, "Autonomous Spectrum Balancing for Digital Subscriber Lines," IEEE Transactions on Signal Processing, vol. 55, no. 8, pp. 4241-4257, 2007.

[3] J. Lee, R. Sonalkar, and J. Cioffi, "Multi-user discrete bit-loading for DMT-based DSL systems," GLOBECOM-NEW YORK-, vol. 2, pp. 1259 1263, 2002.

[4] R. Cendrillon, W. Yu, M. Moonen, J. Verlinden, and T. Bostoen, "Optimal multi-user spectrum management for digital subscriber lines," IEEE Transactions on Communications, vol. 54, no. 5, pp. 922-933, 2006.

[5] R. Cendrillon and M. Moonen, "Iterative spectrum balancing for digital subscriber lines," in 2005 IEEE International Conference on Communications, 2005. ICC 2005, vol. 3, 2005.

[6] J. Papandriopoulos and J. Evans, "Low-complexity distributed algorithms for spectrum balancing in multi-user DSL networks," in IEEE International Conference on Communications, 2006. ICC'06, vol. 7, 2006.

[7] P. N. X. W. Venturino, L., "An Improved Iterative Water-Filling Algorithm for Multi-Cell Interference Mitigation in Downlink OFDMA Networks," in Asilomar Conference on Signals, Systems and Computers, 2007.

[8] A. Fasano, G. Di Blasio, E. Baccarelli, and M. Biagi, "Optimal discrete bit loading for DMT based constrained multicarrier systems," in 2002 IEEE International Symposium on Information Theory, 2002. Proceedings, 2002.

[9] D. Welsh, "Matroid theory," New York, 1976. 